\title{
Bioinformatics Analysis of the Ribosomal Proteins, RPL27, RPL37a and RPL41: 3-D Protein Modeling and Protein-protein Interaction Prediction
}

\author{
Stella L. L. Chan and Edmund U. H. Sim
}

\begin{abstract}
Ribosomal proteins (RPs) are constituents of ribosome important for protein biosynthesis but likely to have extraribosomal functions. Many RPs are associated with various diseases and cancers. A previous study reported RPL27, RPL37a and RPL41 gene to be downregulated in nasopharyngeal carcinoma (NPC) derived cell lines compared to their normal counterpart. However, their actual physiological roles in organogenesis or tumorigenesis have not been properly defined. In this paper, we report on the findings of structural prediction of these three genes and infer their interactions with other proteins using structural neighbor prediction and molecular docking strategies. Our results revealed that RPL27 interact with SYNJ2 and UBC9. RPL27 is predicted to mediate RNA binding protein and deregulate sumolyation. RPL37a is suggested to interact with CTNNB1, SCMH1 and ATBF1. It is predicted to deregulate Wnt degradation pathway, inhibit $\beta$-catenin migration and regulate homeotic transcription. Our studies on RPL41 did not allow logical inference on possible interacting factors. Nevertheless, results on RPL27 and RPL37a provide rational data for the elucidation of their molecular activities.
\end{abstract}

Index Terms-Protein modeling, extraribosomal functions, protein-protein interaction prediction, ribosomal protein.

\section{INTRODUCTION}

Ribosomal proteins (RPs) are important ribosomal subunits assembled for protein biosynthesis. Disruption in this process has been associated with deregulated cell growth and altered cell cycle [1], [2]. Ribosomal proteins are structurally distinct and independent polypeptides [3]. Several of them have been found to possess extraribosomal functions that modulate function of important regulatory proteins. Some can even translationally control their targets outside the ribosome [1], [4].

Ribosomal protein genes have been associated with colorectal carcinoma [5]. Recent studies showed that the ribosomal protein large subunit (RPL) genes of RPL27, RPL37a and RPL41 are significantly downregulated in cell lines derived from nasopharyngeal carcinoma (NPC) compared to a derivative from normal nasopharyngeal epithelium [6], [7]. However, limited studies on protein-protein interaction activities have made the understanding of their extraribosomal roles difficult. One

Manuscript received October 15, 2012; revised December 9, 2012.

Stella L. L. Chan is with the Department of Molecular Biology at Universiti Malaysia Sarawak (UNIMAS), Malaysia (e-mail: still.schan@gmail.com)

Edmund U. H. Sim is with the Department of Molecular Biology, UNIMAS (e-mail: uhsim@frst.unimas.my). reason may be the unavailability of experimental structures due to difficulties in obtaining ribosome component structures [8], [9].

To overcome this problem, our study incorporated bioinformatics approach to understand these three proteins. Three-dimensional (3-D) protein models of these proteins was constructed and served as template to search for structural neighbor.

By the assumption that close homologs or analogs almost always interact in the same way [10], interaction models of queried proteins can be extrapolated from known protein behaviors of their structural neighbors with interacting partners. To strengthen our inferences, RPL models were also docked to their candidate partners to further predict RPL functions through their putative interaction sites. Docking is an amenable method to overcome difficulties in crystalizing and modeling transient complexes [11]. The interaction sites evident from other experiments aid to infer RPL functions. As a result of our endeavor, we revealed novel extraribosomal functions for RPL27 and RPL37a.

\section{Methodology}

\section{A. Multiple sequence alignment}

Protein sequences of human RPL27, RPL37a and RPL41 were retrieved from Ribosomal Protein Gene (RPG) database [12]. The protein identifiers used are: NP_000979 for RPL27, NP_000989 for RPL37a and NP_001030344.1; NP_0667927.1 for RPL41. PSI-BLAST search for each query RPL sequences against Protein Data Bank (PDB) was performed using default parameter to search for templates. For RPL41, the parameter was altered to accommodate short sequence. Template structures with expected value (e-value) above threshold for high sequence identity and structure resolution were selected for multiple sequence alignment. The selected templates were aligned with their respective target sequences using ClustalX program [13]. Protein weight matrix used was from BLOSUM series while clustering algorithm was UPGMA algorithm.

\section{B. Comparative Modeling}

SWISS-MODEL workspace [14] was used to build RPL models. RPL27-template alignments were submitted to SWISS-MODEL server pipeline with 3U5E_Z (PDB ID) as structure template. Using the same alignment mode, RPL37a was modeled with 4A17_Y (PDB ID). In contrast, RPL41 was modeled via project mode by structurally aligning 3IZS (PDB ID) to RPL41 raw protein sequence in SPDBV 4.0.1 
[15]. Quality of the generated models was evaluated by Qualitative Model Energy Analysis (QMEAN6) [16] and PROCHECK [17] from SWISS-MODEL workspace. Models presenting an average QMEAN6 score higher than 0.5 were selected for further analysis.

\section{Protein-protein Interaction Prediction}

The protein models were searched against medium redundancy subset of PDB structures for structural neighbors using Vector Alignment Search Tool (VAST) [18]. The outputs were filtered for human proteins only. The interacting partners to structural neighbors were retrieved from IntAct database [19]. Number of candidate partners for docking was narrowed down based on similarity in sequence patterns using PROSITE search [20].

ClusPro 2.0 server [21] was used to dock RPL model to candidate partners. RPL model was input as receptor and candidate partners as ligand. The returned dock complexes were evaluated based on cluster and energy score. Bound structures to other structures or ligands were excluded for docking. Using SPDBV4.0.1, root mean square distance (r.m.s.d.) was computed when the complex superimposed with their respective unbound structures. Molecular surface of dock complex was scanned for potential interaction sites based on interface contact residues $(5-7 \AA)$.

\section{RESULTS AND DISCUSSION}

\section{A. Analysis of the Multiple Sequence Alignment}

From RPL27sequence alignment (Fig. 1), PfamA search [22] found Kyprides, Woese and Ouzounis (KOW) motif [23] with e-value of $4.1 \mathrm{e}-06$. The motif is in the boundary of residue 7-43 with highest confidence score indicated in grey.

RPL37a alignment (Fig. 2) however, revealed $\mathrm{CX}_{2} \mathrm{CX}_{11,14} \mathrm{CX}_{2} \mathrm{C}$ sequence motif similar to zinc ribbon motif [24]. The motif was characterized by two zinc knuckle motif [25]. Meanwhile, RPL41 alignment shows mainly basic arginine and lysine residues.

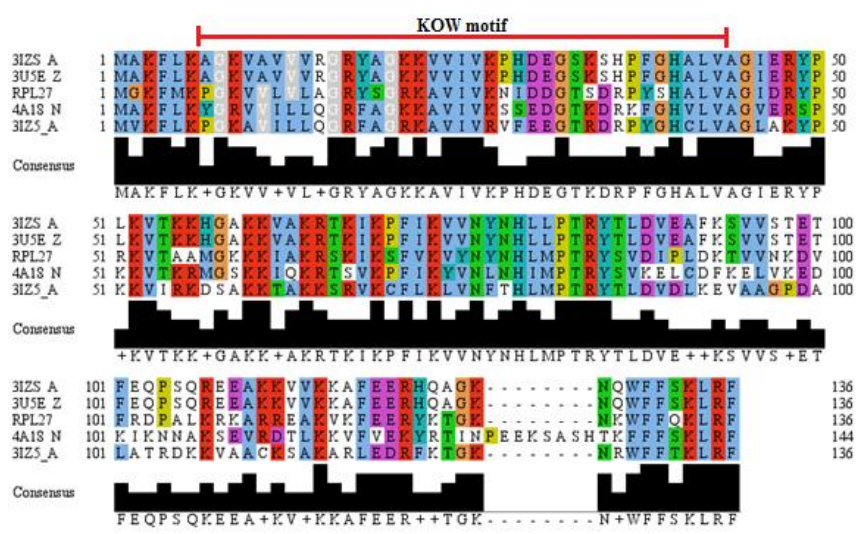

Fig. 1. Multiple sequence alignment of template sequences to RPL27 protein sequence. Figure has been generated using Jalview program [26]. The protein identifiers are as PDB accession number_chain. Alignments are colored using ClustalX scheme (orange: glycine $(\mathrm{G})$; gold: proline (P); blue: small and hydrophobic amino-acids (A, V, L, I, M, F, W); green: hydroxyl and amine amino-acids (S, T, N, Q); magenta: negative-charged amino acids $(\mathrm{D}, \mathrm{E})$; red: positive-charged amino acids $(\mathrm{R}, \mathrm{K})$; dark-blue: histidine $(\mathrm{H})$ and tyrosine $(\mathrm{Y})$ ). Alignments colored grey represented KOW motif. Consensus histogram and sequence were also presented.

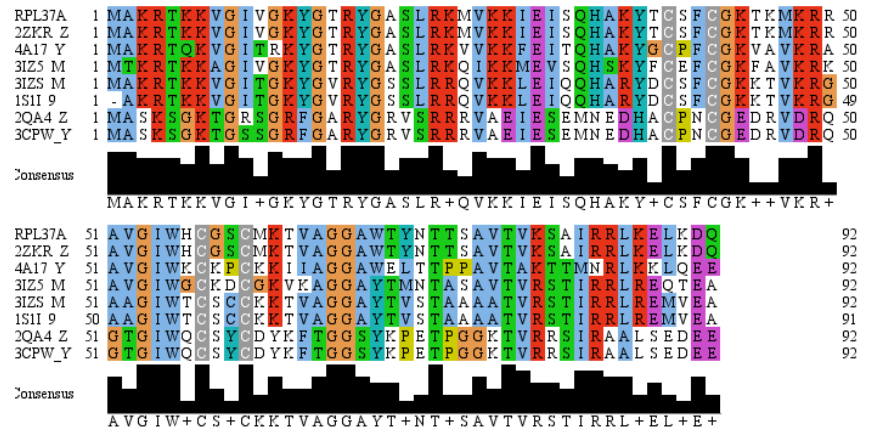

Fig. 2. Multiple sequence alignment of template sequences to RPL37a protein sequence. See caption of figure 1 for details. Highlighted grey residues showed two putative non-canonical zinc finger patterns.

\section{B. 3-D Protein Models of RPL27, RPL37a and RPL41}

RPL27 model (Fig. 3(a)) gives a QMEAN6 score of 0.605 and Z-score of -1.491 (see Table I). PROCHECK, Ramachandran plot analysis shows that $78.3 \%$ of the total residues are in core regions and three residues (Arg17, Lys59 and Arg102) in disallowed high energy regions (see Table II).

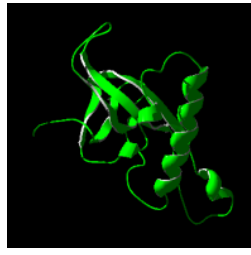

(a)

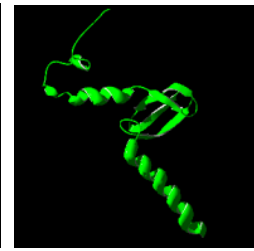

(b)

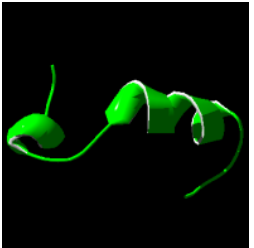

(c)
Fig. 3. (a) RPL27 model (b) RPL37a model (c) RPL41 model presented in ribbon. Ribbon diagrams and other diagrams in this paper were rendered by POV-Ray [27] in SPDBV 4.0.1.

Comparison between RPL27 and Haloarcula marismortui L24 (PDB ID: 1JJ2_S) (r.m.s.d. 1.03 reveals KOW motif embedded as a conserved fold (Fig. 4 (a)). The motif region encompasses the first two strands and their adjacent loops. This motif is most commonly found in microbial L24, eukaryotic RPL26e, RPL27e and bacterial transcription antitermination factor NusG. Moreover, it is mainly implicated in RNA interactions [23]. Nevertheless, the KOW motif was suggested to bind not only to nucleic acids but also to proteins at different surfaces [28].

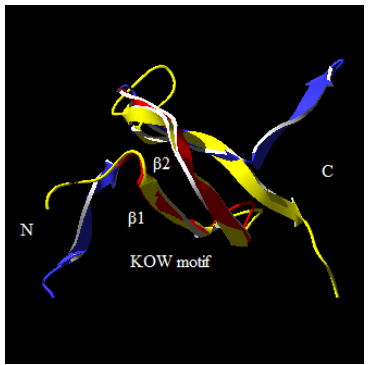

(a)

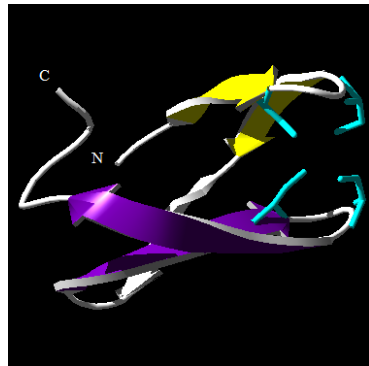

(b)
Fig. 4 (a). Superimposition of RPL27 (yellow) and Haloarcula marismortui L24 (blue). KOW element in L24 was shown in red. (b) Zinc beta ribbon motif in RPL37a model. Primary $\beta$-hairpin is in yellow while secondary in purple. Zinc-knuckles were ball-and-stick presentation of cysteine backbone and side chains (blue)

For RPL37a model (Fig. 3(b)), the QMEAN6 score is 0.501 and -1.944 for $z$-score (see Table I). The Ramachandran plot analysis shows that $91.2 \%$ of the total residues are in the core region (see Table II). When computed for electrostatic potential, the $\mathrm{C}$-terminal helix from residue 
86-92 was detected to be highly negatively charged compared to the rest of the protein. Additionally, zinc beta ribbon motif is distinguished from the three antiparallel $\beta$-strands and two pairing knuckles in the model (Fig. 4 (b)). The two $\beta$-hairpins namely primary and secondary $\beta$-hairpins as well as the conserved cysteine residues form knuckles, presumably for zinc binding.

TABLE I: QMEAN6 SCORES OF RPL27, RPL37A AND RPL41

\begin{tabular}{|c|c|c|c|c|c|c|c|}
\hline Model & $\begin{array}{c}\text { C_beta } \\
\text { interaction } \\
\text { energy }\end{array}$ & $\begin{array}{c}\text { All-atom } \\
\text { pairwise energy }\end{array}$ & $\begin{array}{c}\text { Solvation } \\
\text { energy }\end{array}$ & $\begin{array}{c}\text { Torsion angle } \\
\text { energy }\end{array}$ & $\begin{array}{c}\text { Secondary } \\
\text { structure } \\
\text { agreement }(\%)\end{array}$ & $\begin{array}{c}\text { Solvent } \\
\text { accessibility } \\
\text { agreement }(\%)\end{array}$ & $\begin{array}{l}\text { QMEAN6 } \\
\text { score }\end{array}$ \\
\hline RPL27 & -45.76 & -3127.07 & -12.17 & -20.02 & 75.2 & 71.3 & 0.570 \\
\hline RPL37a & -10.46 & -520.37 & -1.58 & -2.65 & 69.8 & 72.1 & 0.501 \\
\hline RPL41 & 1.43 & -37.72 & -2.24 & 1.30 & 68.0 & 84.0 & 0.588 \\
\hline
\end{tabular}

Q-MEAN6 score is the composite score of all the terms in linear with estimated model reliability between 0-1. C_beta interaction and all-atom pairwise energy refer to two distance-dependent potentials. Solvation potential investigates the burial status of the residues. Torsion angle potential indicates over three consecutive amino acids in local geometry. Secondary structure and solvent accessibility agreement are percentage of agreement between predicted and measured features from sequence to model [29].

TABLE II: PROCHECK ANALYSIS OF RPL27, RPL37A AND RPL41

\begin{tabular}{|c|c|c|c|c|c|c|c|c|c|}
\hline Model & $\begin{array}{c}\text { Core region } \\
(\%)\end{array}$ & $\begin{array}{c}\text { Allowed } \\
\text { region }(\%)\end{array}$ & $\begin{array}{c}\text { Generously } \\
\text { allowed } \\
\text { region }(\%)\end{array}$ & $\begin{array}{l}\text { Disallowed } \\
\text { region }(\%)\end{array}$ & $\begin{array}{c}\text { Bad } \\
\text { contacts }\end{array}$ & G-factor & $\begin{array}{c}\text { M/c bond } \\
\text { lengths }(\%)\end{array}$ & $\begin{array}{l}\mathrm{M} / \mathrm{c} \text { bond } \\
\text { angles }(\%)\end{array}$ & $\begin{array}{c}\text { Planar } \\
\text { region }(\%)\end{array}$ \\
\hline RPL27 & 78.3 & 16.7 & 2.5 & 2.5 & 6 & -0.18 & 100.0 & 95.8 & 93.6 \\
\hline RPL37a & 91.2 & 7.5 & 1.2 & - & - & 0.12 & 100.0 & 97.9 & 100.0 \\
\hline RPL41 & 87.0 & 8.7 & 4.3 & - & - & 0.16 & 100.0 & 97.1 & 100.0 \\
\hline
\end{tabular}

PROCHECK statistical data checks stereochemical quality for RPL27, RPL37a and RPL41 generated from SWISS-MODEL. The different regions correspond to Ramachandran plot [30]. Bad contacts refer to deviation with neighboring residues. G-factors provides measure of how 'normal' or 'unusual' a given stereochemistry property in terms of torsion angles and covalent geometry. $\mathrm{M} / \mathrm{c}$ or main chain bond lengths and angles refer to percentage of agreement to accurate bond and angle parameters. Planar region is percentage of planarity agreement in different planar groups according to r.m.s.d. [17].

RPL41 (Fig. 3 (c)) has a QMEAN6 score of 0.588 and a Z-score of -0.174 (see Table I). Ramachandran plot analysis indicates that $87 \%$ of total residues are in the core regions (see Table II). The final model of RPL27, RPL37a and RPL41 was subjected to docking simulations.

\section{Structural Neighbors and Candidate Partners}

Our results revealed 67 structural neighbors for RPL27 and 48 structural neighbors for RPL37a using VAST. Only RPL41 did not return any result for VAST. RPL41 model has two helices. VAST calculation was not done on protein fewer than three secondary structural elements (SSEs). From IntAct database, RPL27 structural neighbors identified 588 binary protein-protein interactions whereas there are 312 binary interactions for structural neighbors of RPL37a.

In addition, we found matches between known partners of RPL from IntAct database and their VAST structural neighbors. The matches are GABARAPL1 (Uniprot ID: Q9H9R8) and GRB2 (Uniprot ID: P62933) for RPL27; RIF1 (Uniprot ID: Q5UIP0) and PSTPIP1 (Uniprot ID: O43586). This validated our RPL models as functional models.

We excluded all interacting partners with 'spoke' as expansion method. 'Spoke' expansion linked the bait molecule to all prey molecules in co-complex when the real complex might be linked to a smaller complex owing to potential false positives [19]. This allowed us to narrow down our analysis of the hundreds of interacting partners to a few candidate partners based on most similar PROSITE patterns of VAST structural neighbors with RPL model (see Table III). Their degrees of association are from identified recurring signatures, particularly domain which should mediate interactions [31].
TABLE III: SELECTED STRUCTURE NEIGHBorS AND CANDIDATE PARTNERS OF RPL27 AND RPL37A

\begin{tabular}{lcc}
\hline \hline \multirow{2}{*}{ Model } & $\begin{array}{c}\text { Structure neighbor } \\
\text { (PDB ID) }\end{array}$ & $\begin{array}{c}\text { Candidate partners } \\
\text { (PDB ID) }\end{array}$ \\
\hline RPL27 & 1UE9_A & 1UFW \\
& 2E5Q_A & 1A3S \\
RPL37a & 1WJS_A1 & 2Z6H \\
& 2P0K_A2 & 2P0K \\
& 3R6N_A4 & 2DA1 \\
\hline \hline
\end{tabular}

PROSITE that is integrated with ProRule provides functional annotation associated to specific domains. In particular, biological meaningful sites such as, binding sites or active sites [32].

\section{RPL-candidate partner Dock Models}

The ClusPro 2.0 returned top ten dock models for RPL27 and RPL37a. The best of top ten dock models were selected primarily based on biggest cluster size. The model energy scores and r.m.s.d. are fairly consistent (see Table III).

TABLE IV: CLUSPRO SCORES OF RPL-CANDIDATE PARTNER DOCK MODELS

\begin{tabular}{lcccc}
\hline \hline & \multicolumn{4}{c}{ ClusPro Scores } \\
\cline { 2 - 5 } Dock model & $\begin{array}{c}\text { Cluster } \\
\text { size }\end{array}$ & $\begin{array}{c}\text { Center free } \\
\text { energy } \\
(\mathrm{kcal} / \mathrm{mol})\end{array}$ & $\begin{array}{c}\text { Lowest free } \\
\text { energy } \\
(\mathrm{kcal} / \mathrm{mol})\end{array}$ & $\begin{array}{c}\text { r.m.s.d. } \\
(\AA)\end{array}$ \\
\hline RPL27_1UFW & 215 & -758.2 & -941.7 & 1.14 \\
RPL27_1A3S & 201 & -633.3 & -724.4 & 0.94 \\
RPL37a_2Z6H & 59 & -785.0 & -960.5 & 0.82 \\
RPL37a_2P0K & 75 & -675.2 & -803.3 & 1.02 \\
RPL37a_2DA1 & 75 & -477.1 & -625.1 & 1.19 \\
\hline \hline
\end{tabular}

Thus, we predicted protein-protein interaction based on high quality structures of the dock complexes and information 
from mutagenesis and other experiments. Although functional linkage between proteins does not necessarily involve direct physical interactions, binding indicates functional relatedness [10]. Hence, we describe the dock model and their predicted functions postulated from their interactions.

\section{RPL27/SYNJ2 (PDB ID: 1UFW)}

Synaptojanin 2 is a protein involved in recycling of synaptic vesicles by endocytosis with complex of proteins or lipid substrates [33]. However, 1UFW is only the RNA binding protein (RNP) domain of the protein. The four antiparallel $\beta$-sheets or $\beta$-barrel make up the core region important for nucleic acid interaction. From the dock model, only $\beta 1$ of RPL27 KOW motif shows contact $(<5 \AA)$ with a few residues of SYNJ2 $\beta$-barrel (Fig. 5). Despite that, the $\beta$-barrel still has an open-face for other interactions. Evidence of KOW-containing proteins (such as NusG and Spt5) that can mediate ribosome and RNP activities via interaction with latter has been proven in studies by Steiner et al. (2002). This leads us to predict that RPL27 mediates RNP activity.

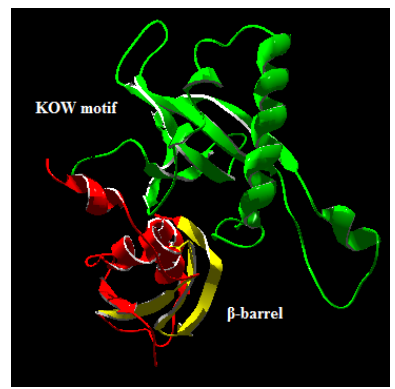

Fig. 5. Protein interaction mode between KOW motif of RPL27 (green) with RNP domain of SYNJ2 (red). The contact still leave core of the domain (yellow) available for other interactions.

\section{RPL27/UBC9 (PDB ID: 1A3S)}

RPL27 docked to small ubiquitin-like modifier (SUMO) -conjugating enzyme E2. SUMO proteins are involved in post-translational modification. Ubiquitin carrier protein 9 (UBC9), in particular, plays an essential role in conjugation of SUMO1 to RanGAP1 [34]. Unlike ubiquinating enzyme, target proteins of SUMO proteins do not lead to degradation by proteasome but their interaction with SUMO proteins leads to a diverse array of functions including subcellular localizations, protein/DNA interactions and enzymatic activities [35]. Sumoylation is carried out by many ribosome biogenesis factors, particularly the preribosomal particles that are found in the small and large ribosomal subunit synthesis pathways. In fact, early stage of large ribosomal subunit biogenesis and export are linked to SUMO conjugation [36].

The UBC9 contains a $\alpha \beta \beta \beta \beta \beta(\beta \beta) \alpha \alpha \alpha$ motif named UBC superfold [37]. From the dock model, UBC9 is shown to interact with Lys 22 and Lys52 (5 ̊) of RPL27 (Fig. 6) which is a characteristic of SUMO protein attachment and modification to substrate [37]. RPL27 is also found to be in contact (7 $\AA$ ) with the conjugation catalytic site of UBC9, Cys93 (Fig. 6). Therefore, we suggest RPL27 as a SUMO target protein. Modification of these lysine residues might either antagonize or stabilize ubiquitin mediated proteolysis such as exhibited by IKB $\alpha$ [38], MDM2 [39], and RPS3 [40].

Besides these, the RPL27-associated UBC9 sites include region important for HECT (Homology to E6AP C-terminus),
RING (Really Interesting New Gene) domain of ubiquitin E3 ligase [41], and secondary binding site for RanGAP1 [42]. The binding site for HECT and RING domain in UBC9 are $\mathrm{N}$-terminal extensions of $\alpha 1$, loops between $\beta 2$ and $\beta 3, \beta 6$ and $\alpha 2$. The $\alpha 3$ catalytic groove of UBC9 is the binding site for RanGAP1, SUMO1, and the UBC12-Nedd8 complex [42]. Competitive binding experiments revealed UBC9 to be an E2 enzyme that dissociate with E1 before formation of E3 complex for sumoylation [43] which strengthens our postulation that RPL27 could deregulate sumolyation through formation of distinct complexes.

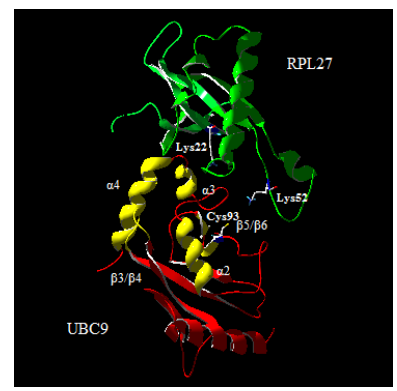

Fig. 6. Protein interaction mode of RPL27(green) with UBC9 (red). Contact region discussed were indicated in yellow while Lys22, Lys52 and catalytic Cys93 were in balls and sticks.

\section{RPL37a/CTNNB1 (PDB ID: 2Z6H)}

Our docking analysis shows association of RPL37a with $\beta$-catenin (CTNNB1). Beta-catenin is a multi-functional protein that plays important roles in adherens junctions and Wnt signaling. Deregulation in Wnt signaling was usually associated with multiple diseases including various cancers [44], [45].

Our docking model reveals that a majority of residues of $\beta$-catenin from the Armadillo (ARM) domains 8-9 are in contact $(<5 \AA)$ with putative zinc ribbon of RPL37a. The ARM domains 11-12 together with C-terminal tail of $\beta$-catenin are in contact with C-terminal helix of RPL37a (Fig. 7). It is plausible that they interact in a dynamic manner due to RPL37a's attraction towards the highly negative $\mathrm{N}$ - and C-termini of $\beta$-catenin. Besides that, the ARM domains 3-8 have been identified to be a hotspot for Tcf-4, E-cadherin, APC and conductin [46]. Meanwhile, ARM domains 5-9 have been reportedly bound to extended groove-binding peptide of E-cadherin [47], several Tcfs [48], APC [49], [50] and ICAT [51], [52]. The grooves of the ARM domains limit their binding to many partners due to steric hindrance [46], [53]. RPL37a might disrupt proper function of Wnt signaling pathway by competing with $\beta$-catenin for binding sites. Other than that, ARM domains 11 and 12 have been shown to interact with ICAT N-terminal helical domain, phosphorylated E-cadherin [47] and APC [49]. These suggest the involvement of RPL37a in the Wnt degradation pathway.

A recent study also indicates ARM domains 10-12 of the C-terminal tail of $\beta$-catenin to be active in the bidirectional transport activity when bound to specific nuclear pore complex (NPC) components [54]. Therefore, we predict that RPL37a might play another role in inhibiting migration of $\beta$-catenin within the cell. Coupled with the RPL37a-mediated deregulation of Wnt degradation complex, $\beta$-catenin level might accumulate in the nucleus which may lead to cell transformation and carcinogenesis [45], [55]. 


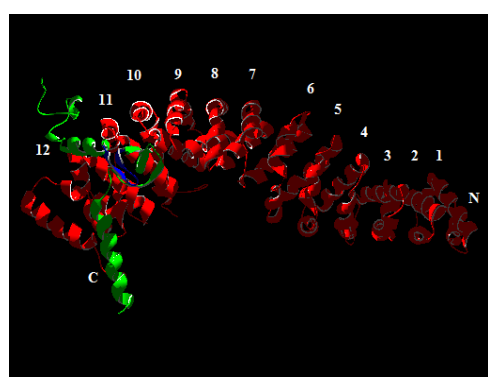

Fig. 7. Protein interaction mode of RPL37a with $\beta$-catenin. RPL37a (green) interact with $\beta$-catenin (red) at zinc ribbon (blue). The numbers represents ARM domains.

\section{RPL37a/SCMH1 (PDB ID: 2P0K)}

Sex comb on midleg homolog 1 (SCMH1) is a repressor required to maintain the silenced state of the homeotic loci. This protein is also a constituent of polycomb repressive complex 1 (Prc 1) [56]. However, the 2P0K protein is only a fragment of SCMH1 which consists of two N-terminal Malignant Brain Tumor (MBT) repeats. The core of this domain is SRC homolog 3 domain (SH3)-like, and comprises a five-stranded $\beta$-barrel that has a C-terminal $\alpha$-helix and a short $\beta$-strand. Although MBT repeat proteins have been shown to recognize methylated lysine residues on histones [57] MBT repeats might function as modules for binding with arginine residues of RPL37a (Fig. 8). A similar situation to this is found in the structurally similar Tudor domain of survival motor neuron protein that binds to methylated arginine residues [58]. However, the overall interface contact between RPL37a and SCMH1 did not involve the zinc ribbon of RPL37a but only MBT2 of SCMH1 (Fig. 8).

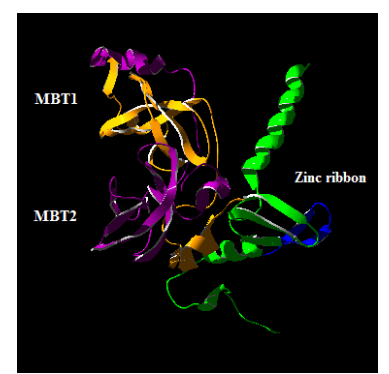

Fig. 8. Protein interaction mode of RPL37a (green) and SCMH1. Zinc ribbon is in blue, MBT1 in yellow while MBT in purple.

\section{RPL37a/ATBF1 (PDB ID: 2DA1)}

2DA1 is solution structure of the first homeobox domain of AT-binding transcription factor 1 (ATBF1). It is a homeotic transcriptional regulatory factor that acts as a tumor suppressor [59]. The homeodomain consists of helix-turn-helix (HTH) fold with a hydrophobic core. Homeodomain is also known to make contact with DNA through C-terminal end of a third helix. In our case, the C-terminal tail of ATBF1 makes contact with $\alpha 1$ and C-terminal peptides of RPL37a (Fig. 9).

Homeodomain-containing transcription factors often interact with other transcription factors and coactivators to enhance transcription activity by binding to the site adjacent to HTH motif [60]. Therefore, we predict that RPL37a regulates homeotic transcription by competing with other transcription factors and coactivators for binding sites within the homeodomain of ATBF1.

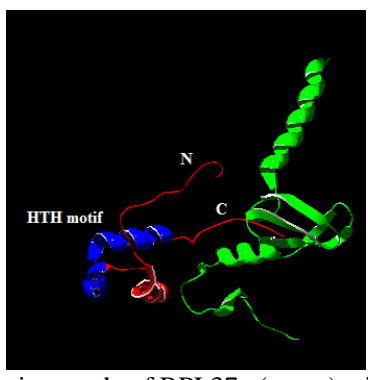

Fig. 9. Protein interaction mode of RPL37a (green) with ATBF1 (red). The helix-turn-helix fold of ATBF1 is in blue.

\section{CONCLUSION}

Comparative modeling is useful and capable of generating functional models for further analysis. In our case, the RPL27 and RPL37a 3-D models allowed us to identify structural neighbors via VAST analysis. Selected structural neighbors assigned candidate partners for docking to predict protein-protein interaction and functions of the RPL proteins studied. Our findings revealed novel plausible functions of these proteins. For example, RPL27 might mediate RNP and deregulate sumolyation whereas RPL37a might be involved in Wnt degradation pathway, inhibits $\beta$-catenin migration and regulate homeotic transcription. Information such as these will provide reference knowledge to design experiments that can accurately delineate the definitive physiological roles of RPL27 and RPL37a.

\section{REFERENCES}

[1] D. Ruggero, and P. P. Pandolfi, "Does the ribosome translate cancer?" Nature Reviews, vol. 3, pp. 179-192, March 2003

[2] J. R. Warner, and K. B. McIntosh, "How common are extraribosomal functions of ribosomal proteins?" Molecular Cell, vol. 34, pp. 3-11, April 2009.

[3] K. Ishii, T. Washio, T. Uechi, M. Yoshihama, N. Kenmochi et al., "Characteristics and clustering of human ribosomal protein genes," BMC Genomics, vol. 7, pp. 37-52, February 2006.

[4] M. S. Lindström, "Emerging functions of ribosomal proteins in gene specific transcription and translation," Biochemical and Biophysical Research Communications, vol. 379, pp. 167-170, December 2008.

[5] E. U. H. Sim, I. P. N. Bong, P. Balraj, S. K. Tan, R. Jamal et al., "A preliminary study of differential expressed genes in Malaysian colorectal carcinoma cases," Journal of Biosciences, vol. 17, pp. 19-37, 2006.

[6] E. U. H. Sim, A. K. L. Toh, and T. S. Tiong, "Preliminary findings of downregulated genes in nasopharyngeal carcinoma," Asia Pacific Journal of Molecular Biology \& Biotechnology, vol. 16, pp. 79-84, 2008.

[7] E. U. H. Sim, C. H. Ang, C. C. Ng, C. W. Lee, and K. Narayanan. "Differential expression of a subset of ribosomal protein genes in cell lines derived from human nasopharyngeal epithelium," Journal of human genetics, vol. 55, pp. 118-120, 2010.

[8] D. J. Klein, P. B. Moore, and T. A. Steitz, "The roles of ribosomal proteins in the structure assembly, and evolution of the large ribosomal subunit," J. Mol. Biol., vol. 340, pp. 141-177, 2004.

[9] T. M. Schmeing, and V. Ramakrishnan, "What recent ribosome structures have revealed about the mechanism of translation," Nature, vol. 461, pp. 1243-1242, 2009.

[10] A. Szilágyi, V. Grimm, A. Arakaki, and J. Skolnick, "Prediction of physical protein-protein interactions," Phys. Biol., vol. 2, pp. S1-16, 2005.

[11] I. Ezkurdia, L. Bartoli, P. Fariselli, R. Casadio, A. Valencia, and M. L. Tress, "Progress and challenges in predicting protein-protein interaction sites," Briefings in Bioinformatics, vol. 10, pp. 233-246, April 2009.

[12] A. Nakao, M. Yoshihama, and N. Kenmochi, "RPG: the Ribosomal Protein Gene database," Nucl. Acids Res., vol. 32, pp. 169-170, 2004. 
[13] M. A. Larkin, G. Blackshields, N. P. Br.own, R. Chenna, P. A. McGettigan et al., "Clustal W and Clustal X version 2.0," Bioinformatics, vol. 23, pp. 2947-2948, 2007.

[14] T. Schwede, J. Koop, N. Guex, and M. C. Peitsch, "SWISS-MODEL: an automated protein homology-modeling server," Nucl. Acids. Res., vol. 31, pp3381-3385, 2003.

[15] N. Guex, and M. C. Peitsch, "SWISS-MODEL and the Swiss-Pdb Viewer: an environment for comparative protein modeling," Electrophoresis, vol. 18, pp. 2714-2723, 1997.

[16] P. Benkert, M. Kuenzli, and T. Schwede, "QMEAN server for protein model quality estimation," Nucl. Acids. Res., vol. 37, pp. W510-W514, 2009.

[17] R. A. Laskowski, M. A. MacArthur, D. S. Moss, and J. M. Thornton, "PROCHECK: a program to check stereochemical quality of protein structures," J. Appl. Cryst, vol. 26, pp. 283-291, 1993.

[18] J. F. Gibrat, T. Madej, and S. H. Bryant, "Suprising similarities in structure comparison," Curr. Opin. Struct. Biol., vol. 6, pp. 377-385, June 1996

[19] S. Kerrien, B. Aranda, I. Breuza, A. Bridge, F. Broackes-Carter et al., "The IntAct molecular interaction database in 2012," Nucl. Acids. Res. vol. 40, pp. D841-D846, 2012.

[20] N. Hulo, A. Bairoch, V. Bulliard, L. Cerutti, E. De Castro et al., "The PROSITE database," Nucl. Acids. Res., vol. 34, pp. D227-D230, 2006.

[21] S. R. Comeau, D. W. Gatchell, S. Vajda, and C. J. Camacho, "ClusPro: a fully automated algorithm for protein-protein docking," Nucl. Acids. Res., vol. 32, pp. W96-W99, February 2004.

[22] M. Punta, P. C. Coggill, R. Y. Eberhadt, J. Mistry, J. Tate et al., “ The Pfam protein families database," Nucl. Acids. Res., vol. 40, pp. D290-D301, 2012.

[23] N. C. Kyprides, C. R. Woese, and C. A. Ouzounis, "KOW: a novel motif linking a bacterial transcription factor with ribosomal proteins," Trends. Biochem. Sci., vol. 21, pp. 425-426, 1996.

[24] J. H, Laity, B. M. Lee, and P. E. Wright, "Zinc finger proteins: new insights into structural and functional diversity," Curr. Opin. Struc. Bio., vol. 11, pp. 39-46, 2001.

[25] S. S. Krishna, I. Majumdar, and N. V. Grishin, "Structural classification of zinc fingers," Nucl. Acids. Res., vol. 31, pp. 532-550, 2003.

[26] M. Clamp, J. Cuff, S. M. Searle, and G. J. Barton, "The Jalview Java alignment editor," Bioinformatics, vol. 20., pp. 427-427, 2004.

[27] Persistence of Vision Pty. Ltd. (2004). On the use of Persistence of Vision Raytracer. (Version 3.6). [Online]. Available: http://www.povray.org/download/

[28] T. Steiner, J. T. Kaiser, S. Marinkoviç, and M. C. Wahl, "Crystal structures of transcription factor NusG in light of its nucleic acid and protein-binding activities," EMBO J., vol. 21, pp. 4641-4653, 2002.

[29] P. Benkert, S. C. E. Tosatto, and D. Schomburg, "QMEAN: A comprehensive scoring function for model quality assessment," Proteins: Structure, Function and Bioinformatics, vol. 71, p. 261-277, 2008.

[30] A. L. Morris, M. W. MacArthur, E. G. Hutchinson, and J. M. Thornton, "Assessing the accuracy of protein structural coordinates," Proteins: Structure, Function and Bioinformatics, vol. 12, pp. 345-364, 1992.

[31] M. Deng, S. Mehta, F. Sun, and T. Chen, "Inferring domain-domain interactions from protein-protein interactions," Genome Res., vol. 12, pp. 1540-1548, 2002.

[32] C. J. A. Sigrist, L. Cerutti, E. De Castro, P. S. Langendijk-Genevaux, V. Bulliard et al., "PROSITE: a protein domain database for functional characterization and annotation," Nucl. Acids. Res., vol. 38, pp. D161D166, 2009.

[33] T. W. Harris, E. Hartweig, H. R. Hotviz, and E. M. Jorgensen, "Mutations in sypnatojanin disrupt sypnatic vesicle recycling," J. Cell. Biol., vol. 150, pp. 589-600, 2000.

[34] M. J. Matunis, J. Wu, and G. Blobel, "SUMO-1 modification and its role in targeting the Ran GTPase- activating protein, RanGAP1, to the nuclear pore complex," J. Cell. Biol., vol. 140, pp.499-509, 1998.

[35] F. Melchior, "SUMO-non classical ubiquitin," Annual Review of Cell and Developmental Biology, vol. 18, pp. 591-626, 2000.

[36] V. G. Panse, A. Kressler, E. Pauli, M. Petfalski, D. Gnadig et al., "Formation and nuclear export of preribosomes are functionally linked to the small-ubiquitin-related modifier pathway," Traffic, vol. 7, pp.1131-1321, 2006

[37] Z. Tang, C. M. Hecker, A. Scheschonka, and H. Betz, "Protein interactions in the sumoylation cascade-lessons from X-ray structures," FEBS J., vol. 275, pp.3003-3015, 2008.
[38] J. M. Desterro, M. S. Rodriguez, and R. T. Hay, "SUMO-1 modification of IkappaBalpha inhibits NF-kappaB activation," Mol. Cell., vol. 2, pp. 233-239, 1998.

[39] T. Buschmann, S. Y. Fuchs, C. G. Lee, Z. Q. Pan, and Z. Ronai, "SUMO-1 modificaiton of Mdm2 prevents its self-ubiquination and increases Mdm2 ability to ubiquinate p53," Cell, vol. 101, pp.753-762, 2000.

[40] C. Y. Jang, H. S. Shin, H. D. Kim, J. W. Kim, S. Y. Choi et al., "Ribosomal protein S3 in stabilized by sumoylation," Biochem. Biophys. Res. Commun., vol. 414, pp.523-527, September 2011.

[41] N. Zheng, P. Wang, P. D. Jeffrey, and N. P. Pavletich, "Structure of a c-Cbl-UbcH7 complex: RING domain function in ubiquitin -protein ligases," Cell, vol. 102, pp. 533-539, 2000.

[42] D. Reverter, and C. D. Lima, "Insights into E3 ligase activity revealed by a SUMO-RanGAP1-Ubc9-Nup358 complex," Nature, vol. 435, pp. 687-692, 2005.

[43] B. T. Dye, and B. A. Schulman, "Structural mechanisms underlying posttranslational modification by ubiquitin-like proteins," Anпи. Rev. Biophys. Struct. , vol. 36, pp. 31-150, 2007.

[44] T. Reya, and H. Clevers, "Wnt signaling in stem cells and cancer" Nature, vol. 434, pp.843-850, 2005.

[45] P. Polakis, "Wnt signaling and cancer," Genes Dev., vol. 14, pp.1837-18 51, 2000.

[46] J. P. Von Kries, G. Winbeck, C. Asbrand, T. Schwarz-Romond, N Sochnikova et al., "Hotspots in $\beta$-catenin for interactions with LEF-1, conductin and APC," Nature, vol. 7, pp.800-807, 2000.

[47] A. H. Huber, W. I. Weis, "The structure of $\beta$-catenin/E-cadherin complex and the molecular basis of diverse ligand recognition by $\beta$-catenin," Cell, vol. 105, pp.391-402, 2001.

[48] F. Poy, M. Lepourcelet, R. A. Shivdasani, and M. J. Eck, "Structure of a human Tcf-4ß-catenin complex," Nat. Struct. Biol., vol. 8, pp. 1053-1057, 2001.

[49] Y. Xing, W. K. Clements, L. Le Trong, T. R. Hinds, R. Stenkamp et al., "Crystal structure of a $\beta$-catenin/APC complex reveals a critical role for APC phosphorylation in APC function," Mol. Cell., vol. 15, pp.523-533, 2004.

[50] N. C. Ha, T. Tonozuka, J. L. Stamos, H. J. Choi, and W. I. Weis, "Mechanism of phosphorylation-dependent binding of APC to $\beta$-catenin degradation," Mol. Cell., vol. 15, pp.511-521, 2004.

[51] D. L. Daniels, and W. I. Weis, "ICAT inhibits $\beta$-catenin binding to Tcf/Lef-family transcription factors and the general coactivator p300 using independent structural modules," Mol. Cell., vol. 10, pp. 573-584, 2002.

[52] T. A. Graham, W. K. Clements, D. Kimelman, and W. Xu, "The crystal structure of $\beta$-catenin/ICAT complex reveals the inhibitory mechanism of ICAT," Mol. Cell., vol. 10, pp. 563-571, 2002.

[53] H. Choi, A. H. Huber, and W. I. Weis, "Thermodynamics of $\beta$-catenin-ligand interactions," Journal of Biological Chemistry, vol. 281, pp.1027-1038, 2006.

[54] M. Sharma, C. Jamieson, M. Johnson, M. P. Molloy, and B. R. Henderson, "Specific armadillo repeat sequences facilitate $\beta$-catenin nuclear transport in live cells via direct binding to nucleoporins Nup62, Nup 153, and RanBP2/Nup358," J. Biol. Chem., vol. 287, pp.819-831, 2012.

[55] R. T. Moon, B. Bowerman, M. Boutros, and N. Perrimon, "The promise and perils of Wnt signaling through $\beta$-catenin" Science, vol. 296, pp. 1644-1646, 2002.

[56] J. Berger, H. Kurahashi, Y. Takihara, K. Shimada, H. W. Brock et la. "The human homolog of Sex comb on midleg (SCMH1) maps to chromosome 1p34," Gene, vol. 237, pp. 185-191, 1999.

[57] J. Kim, J. Daniel, A. Lake, M. Krishna et al., "Tudor, MBT and chromo domains gauge the degree of lysine methylation," EMBO Rep., vol. 4, pp. 397-403, April 2006.

[58] P. Selenko, R. Sprangers, G. Stier, D. Bühler, U. Fischer et al., "SMN tudor domain structure and its interaction with the Sm proteins," Nat. Struct. Biol, vol. 8, pp.27-31, 2001.

[59] Y. Mori, H. Kaaoka, Y. Miura, M. Kawaguchi, E. Kubota et al, "Subcellular localization of ATBF1 regulates MUC5AC transcription in gastric cancer," Int. J. Cancer, vol. 121, pp. 241-247, 2007.

[60] T. Di Palma, R. Nitsch, A. Mascia, L. Nitsch, R. Di Lauro et al., "The paired domain-containing factor Pax8 and the homeodomain-containing factor TTF-1 directly interact and synergistically activate transcription," J. Biol. Chem., vol. 278, pp. $3395-3402,2002$. 